\title{
SUPPLEMENTARY INFORMATION (SI) FILE
}

Prebiotic efficacy of oligosaccharides derived from the immunomodulatory glycoprotein (Agp) purified from ajowan (Trachyspermum ammi L.) DOI: https://doi.org/10.1021/acsfoodscitech.1c00078

Raju R. Shruthi, Yeldur P. Venkatesh and Muralikrishna Gudipati*

Department of Biochemistry, CSIR-Central Food Technological Research Institute (CSIRCFTRI), Mysore, Karnataka, India-570020

*Corresponding author

Dr. G. Muralikrishna Chief Scientist (Retd.,),

Formerly HOD and Chief Scientist, Department of Biochemistry, CSIR-CFTRI, KRS Road, Mysore 570020, Karnataka state, India.

E-mail: krishnagm2002@yahoo.com

Tel: +919480382199 
Table ST1 (a to c). Growth characteristics of selected microorganisms in the presence of Agp-oligosaccharides.

(a) Growth of L. delbrueckii (NCDC 10) with different concentrations of Agpoligosaccharides

\section{Absorbance at 600 nm}

\begin{tabular}{|c|c|c|c|c|c|}
\hline Time (h) & $\mathbf{0}$ & 24 & 48 & 72 & 96 \\
\hline Control & $0.10 \pm 0.01$ & $0.11 \pm 0.03$ & $0.23 \pm 0.01$ & $21 \pm 0.03$ & $0.20 \pm 0.04$ \\
\hline $\begin{array}{l}\text { Agp-oligosaccharides } \\
(0.25 \%)\end{array}$ & $0.09 \pm 0.02$ & $0.58 \pm 0.01$ & $0.63 \pm 0.02$ & $61 \pm 0.07$ & $0.60 \pm 0.02$ \\
\hline $\begin{array}{l}\text { Agp-oligosaccharides } \\
(0.5 \%)\end{array}$ & $0.11 \pm 0.01$ & $0.61 \pm 0.02$ & $0.65 \pm 0.01$ & $65 \pm 0.09$ & $0.63 \pm 0.02$ \\
\hline $\begin{array}{l}\text { Agp-oligosaccharides } \\
(1 \%)\end{array}$ & $0.09 \pm 0.02$ & $0.63 \pm 0.01$ & $0.77 \pm 0.05$ & $70 \pm 0.03$ & $0.71 \pm 0.03$ \\
\hline \multirow[t]{2}{*}{ FOS $(0.5 \%)$} & $0.08 \pm 0.01$ & $0.89 \pm 0.06$ & $0.91 \pm 0.04$ & $92 \pm 0.03$ & $0.91 \pm 0.05$ \\
\hline & \multicolumn{5}{|c|}{ pH of the culture media } \\
\hline Time (h) & 0 & 24 & 48 & 72 & 96 \\
\hline Control & $7.0 \pm 0.1$ & $7.0 \pm 0.1$ & $6.9 \pm 0.3$ & $6.9 \pm 0.3$ & $7.1 \pm 0.4$ \\
\hline $\begin{array}{l}\text { Agp-oligosaccharides } \\
(0.25 \%)\end{array}$ & $6.9 \pm 0.1$ & $6.8 \pm 0.2$ & $6.3 \pm 0.4$ & $6.3 \pm 0.3$ & $6.2 \pm 0.3$ \\
\hline $\begin{array}{l}\text { Agp-oligosaccharides } \\
(0.5 \%)\end{array}$ & $6.8 \pm 0.1$ & $6.7 \pm 0.4$ & $6.1 \pm 0.6$ & $6.2 \pm 0.4$ & $6.1 \pm 0.4$ \\
\hline $\begin{array}{l}\text { Agp-oligosaccharides } \\
(1 \%)\end{array}$ & $6.9 \pm 0.1$ & $6.6 \pm 0.2$ & $5.8 \pm 0.3$ & $5.8 \pm 0.2$ & $5.4 \pm 0.2$ \\
\hline \multirow[t]{2}{*}{ FOS $(0.5 \%)$} & $6.8 \pm 0.1$ & $5.7 \pm 0.5$ & $5.3 \pm 0.4$ & $5.2 \pm 0.2$ & $5.2 \pm 0.5$ \\
\hline & \multicolumn{5}{|c|}{ Dry cell mass (mg/mL broth) } \\
\hline Time (h) & 0 & 24 & 48 & 72 & 96 \\
\hline Control & $0.004 \pm 0.001$ & $0.08 \pm 0.002$ & $0.091 \pm 0.001$ & $0.092 \pm 0.01$ & $0.08 \pm 0.010$ \\
\hline $\begin{array}{l}\text { Agp-oligosaccharides } \\
(0.25 \%)\end{array}$ & $0.004 \pm 0.002$ & $0.36 \pm 0.03$ & $0.65 \pm 0.03$ & $0.66 \pm 0.04$ & $0.62 \pm 0.01$ \\
\hline $\begin{array}{l}\text { Agp-oligosaccharides } \\
(0.5 \%)\end{array}$ & $0.003 \pm 0.001$ & $0.83 \pm 0.05$ & $1.30 \pm 0.02$ & $1.32 \pm 0.03$ & $1.31 \pm 0.03$ \\
\hline $\begin{array}{l}\text { Agp-oligosaccharides } \\
(1 \%)\end{array}$ & $0.004 \pm 0.001$ & $0.92 \pm 0.04$ & $1.20 \pm 0.05$ & $1.22 \pm 0.02$ & $1.21 \pm 0.05$ \\
\hline FOS $(0.5 \%)$ & $0.004 \pm 0.001$ & $0.99 \pm 0.01$ & $1.23 \pm 0.03$ & $1.32 \pm 0.02$ & 1.360 .03 \\
\hline
\end{tabular}


(b) Growth of L. acidophilus (NCDC 11) with different concentrations of Agpoligosaccharides

\begin{tabular}{|c|c|c|c|c|c|}
\hline \multirow[b]{2}{*}{ Time (h) } & \multicolumn{5}{|c|}{ Absorbance at $600 \mathrm{~nm}$} \\
\hline & 0 & 24 & 48 & 72 & 96 \\
\hline Control & $0.04 \pm 0.002$ & $0.09 \pm 0.003$ & $0.14 \pm 0.04$ & $0.19 \pm 0.03$ & $0.11 \pm 0.01$ \\
\hline $\begin{array}{l}\text { Agp-oligosaccharides } \\
(0.25 \%)\end{array}$ & $0.02 \pm 0.001$ & $0.68 \pm 0.04$ & $0.91 \pm 0.04$ & $0.91 \pm 0.03$ & $0.90 \pm 0.05$ \\
\hline Agp-oligosaccharides $(0.5 \%)$ & $0.02 \pm 0.002$ & $0.76 \pm 0.03$ & $0.89 \pm 0.05$ & $0.91 \pm 0.03$ & $0.90 \pm 0.02$ \\
\hline Agp-oligosaccharides (1\%) & $0.03 \pm 0.001$ & $0.81 \pm 0.03$ & $0.89 \pm 0.01$ & $0.91 \pm 0.01$ & $0.90 \pm 0.04$ \\
\hline \multirow[t]{2}{*}{ FOS $(0.5 \%)$} & $0.02 \pm 0.001$ & $0.86 \pm 0.02$ & $0.93 \pm 0.03$ & $0.93 \pm 0.02$ & $0.92 \pm 0.02$ \\
\hline & \multicolumn{5}{|c|}{ pH of the culture media } \\
\hline Time (h) & 0 & 24 & 48 & 72 & 96 \\
\hline Control & $6.7 \pm 0.1$ & $7.1 \pm 0.3$ & $6.9 \pm 0.2$ & $7.1 \pm 0.3$ & $6.9 \pm 0.1$ \\
\hline $\begin{array}{l}\text { Agp-oligosaccharides } \\
(0.25 \%)\end{array}$ & $6.7 \pm 0.1$ & $6.6 \pm 0.1$ & $6.2 \pm 0.2$ & $5.9 \pm 0.5$ & $5.8 \pm 0.2$ \\
\hline Agp-oligosaccharides $(0.5 \%)$ & $6.6 \pm 0.2$ & $6.1 \pm 0.4$ & $5.7 \pm 0.3$ & $5.8 \pm 0.3$ & $5.8 \pm 0.3$ \\
\hline Agp-oligosaccharides (1\%) & $6.6 \pm 0.1$ & $6.4 \pm 0.5$ & $5.7 \pm 0.4$ & $5.6 \pm 0.2$ & $5.6 \pm 0.4$ \\
\hline \multirow[t]{2}{*}{ FOS $(0.5 \%)$} & $6.6 \pm 0.2$ & $5.5 \pm 0.2$ & $5.5 \pm 0.3$ & $5.4 \pm 0.2$ & $5.5 \pm 0.2$ \\
\hline & \multicolumn{5}{|c|}{ Dry cell mass (mg/mL broth) } \\
\hline Time (h) & 0 & 24 & 48 & 72 & 96 \\
\hline Control & $0.004 \pm 0.002$ & $0.09 \pm 0.002$ & $0.098 \pm 0.01$ & $0.10 \pm 0.011$ & $0.09 \pm 0.01$ \\
\hline $\begin{array}{l}\text { Agp-oligosaccharides } \\
(0.25 \%)\end{array}$ & $0.005 \pm 0.001$ & $0.65 \pm 0.06$ & $0.71 \pm 0.05$ & $0.72 \pm 0.04$ & $0.73 \pm 0.03$ \\
\hline $\begin{array}{l}\text { Agp-oligosaccharides } \\
(0.5 \%)\end{array}$ & $0.004 \pm 0.001$ & $0.77 \pm 0.04$ & $0.98 \pm 0.05$ & $1.02 \pm 0.02$ & $1.03 \pm 0.03$ \\
\hline $\begin{array}{l}\text { Agp-oligosaccharides } \\
(1 \%)\end{array}$ & $0.005 \pm 0.001$ & $0.82 \pm 0.05$ & $0.99 \pm 0.07$ & $1.04 \pm 0.04$ & $1.02 \pm 0.02$ \\
\hline FOS $(0.5 \%)$ & $0.005 \pm 0.001$ & $0.98 \pm 0.08$ & $1.11 \pm 0.06$ & $1.12 \pm 0.03$ & $1.10 \pm 0.05$ \\
\hline
\end{tabular}


(c) Growth of L. casei (NCDC 17) with different concentrations of Agpoligosaccharides

\begin{tabular}{|c|c|c|c|c|c|}
\hline \multicolumn{6}{|c|}{ Absorbance at $600 \mathrm{~nm}$} \\
\hline Time (h) & 0 & 24 & 48 & 72 & 96 \\
\hline Control & $0.06 \pm 0.006$ & $0.12 \pm 0.009$ & $0.11 \pm 0.02$ & $0.13 \pm 0.01$ & $0.12 \pm 0.02$ \\
\hline $\begin{array}{l}\text { Agp-oligosaccharides } \\
(0.25 \%)\end{array}$ & $0.01 \pm 0.002$ & $0.62 \pm 0.05$ & $0.86 \pm 0.02$ & $0.83 \pm 0.05$ & $0.84 \pm 0.02$ \\
\hline $\begin{array}{l}\text { Agp-oligosaccharides } \\
(0.5 \%)\end{array}$ & $0.009 \pm 0.001$ & $0.59 \pm 0.05$ & $0.87 \pm 0.01$ & $0.91 \pm 0.05$ & $0.90 \pm 0.04$ \\
\hline $\begin{array}{l}\text { Agp-oligosaccharides } \\
(1 \%)\end{array}$ & $0.01 \pm 0.001$ & $0.71 \pm 0.06$ & $0.78 \pm 0.04$ & $0.82 \pm 0.04$ & $0.81 \pm 0.03$ \\
\hline FOS $(0.5 \%)$ & $0.008 \pm 0.001$ & $0.83 \pm 0.02$ & $0.85 \pm 0.05$ & $0.93 \pm 0.03$ & $0.92 \pm 0.05$ \\
\hline \multicolumn{6}{|c|}{ pH of the culture media } \\
\hline Time (h) & 0 & 24 & 48 & 72 & 96 \\
\hline Control & $6.8 \pm 0.1$ & $7.1 \pm 0.2$ & $6.8 \pm 0.4$ & $6.9 \pm 0.2$ & $6.8 \pm 0.4$ \\
\hline $\begin{array}{l}\text { Agp-oligosaccharides } \\
(0.25 \%)\end{array}$ & $6.7 \pm 0.2$ & $6.7 \pm 0.3$ & $6.1 \pm 0.2$ & $6.2 \pm 0.5$ & $6.2 \pm 0.2$ \\
\hline $\begin{array}{l}\text { Agp-oligosaccharides } \\
(0.5 \%)\end{array}$ & $6.6 \pm 0.1$ & $6.1 \pm 0.2$ & $5.6 \pm 0.4$ & $5.4 \pm 0.4$ & $5.5 \pm 0.5$ \\
\hline $\begin{array}{l}\text { Agp-oligosaccharides } \\
(1 \%)\end{array}$ & $6.6 \pm 0.2$ & $5.9 \pm 0.4$ & $5.8 \pm 0.3$ & $5.8 \pm 0.1$ & $5.7 \pm 0.3$ \\
\hline FOS $(0.5 \%)$ & $6.7 \pm 0.1$ & $5.7 \pm 0.3$ & $5.4 \pm 0.3$ & $5.4 \pm 0.3$ & $5.5 \pm 0.3$ \\
\hline & \multicolumn{5}{|c|}{ Dry cell mass (mg/mL broth) } \\
\hline Time (h) & 0 & 24 & 48 & 72 & 96 \\
\hline Control & $0.007 \pm 0.001$ & $0.087 \pm 0.005$ & $0.086 \pm 0.003$ & $0.089 \pm 0.002$ & $0.09 \pm 0.006$ \\
\hline $\begin{array}{l}\text { Agp-oligosaccharides } \\
(0.25 \%)\end{array}$ & $0.006 \pm 0.002$ & $0.59 \pm 0.07$ & $0.59 \pm 0.03$ & $0.61 \pm 0.05$ & $0.60 \pm 0.06$ \\
\hline $\begin{array}{l}\text { Agp-oligosaccharides } \\
(0.5 \%)\end{array}$ & $0.004 \pm 0.001$ & $0.78 \pm 0.03$ & $0.88 \pm 0.05$ & $0.91 \pm 0.08$ & $0.92 \pm 0.07$ \\
\hline $\begin{array}{l}\text { Agp-oligosaccharides } \\
(1 \%)\end{array}$ & $0.006 \pm 0.002$ & $0.85 \pm 0.05$ & $0.94 \pm 0.08$ & $0.95 \pm 0.06$ & $0.95 \pm 0.08$ \\
\hline FOS $(0.5 \%)$ & $0.006 \pm 0.001$ & $0.91 \pm 0.07$ & $0.99 \pm 0.08$ & $1.06 \pm 0.04$ & $1.07 \pm 0.07$ \\
\hline
\end{tabular}


Table ST2. NMR $\left({ }^{1} \mathrm{H}\right.$ and $\left.{ }^{13} \mathrm{C}\right)$ spectra of purified Agp-oligosaccharide I (703.88 Da)

\begin{tabular}{|c|c|c|c|c|c|c|}
\hline \multirow{2}{*}{$\begin{array}{c}\text { Glycosidic linkage } \\
{ }^{1} \mathrm{H} \text { NMR }\end{array}$} & \multicolumn{6}{|c|}{ Chemical shifts (ppm) } \\
\hline & H-1 & H-2 & H-3 & H-4 & H-5 & H-6 \\
\hline$\beta$-D Gal P-terminal & 4.44 & 3.52 & 3.63 & 3.92 & 3.70 & 3.90 \\
\hline 3,6-linked & 4.52 & 3.66 & 3.75 & 4.13 & 3.92 & 3.94 \\
\hline 3-linked & 4.49 & 3.55 & 3.61 & 4.10 & 3.70 & 3.81 \\
\hline uronic acid & 4.52 & 3.37 & 3.52 & 3.55 & 3.75 & - \\
\hline${ }^{13} \mathrm{C}$ NMR & C-1 & $\mathrm{C}-2$ & $\mathrm{C}-3$ & $\mathrm{C}-4$ & $\mathrm{C}-5$ & C-6 \\
\hline$\beta$-D Gal P-terminal & 103.55 & 73.84 & 75.13 & 71.41 & 75.24 & 71.85 \\
\hline 3,6-linked & 104.12 & 72.70 & 82.15 & 71.04 & 75.62 & 71.2 \\
\hline 3-linked & 104.36 & 75.62 & 76.01 & 73.77 & 76.02 & - \\
\hline Uronic acid & 104.30 & 75.60 & 76.20 & 73.80 & 82.50 & - \\
\hline
\end{tabular}

Table ST3. NMR $\left({ }^{1} \mathrm{H}\right)$ spectra of purified Agp-oligosaccharide II (687.93 Da)

Glycosidic linkage

\begin{tabular}{ccccccc}
\hline 1H NMR & H-1 & H-2 & H-3 & H-4 & H-5 & H-6 \\
\hline a-L Araf terminal & 5.2 & 4.00 & 3.93 & 4.40 & 3.84 & - \\
\hline Araf $\boldsymbol{\alpha - 1 - 3}$ & 5.00 & 4.22 & 3.95 & 4.00 & 3.38 & - \\
\hline Gal 3, 6-linked & 4.52 & 3.65 & 3.75 & 4.00 & 3.92 & 4.00 \\
\hline oAc group & $2-3$ & & & & & \\
\hline
\end{tabular}




\section{Supplementary figures}

Figure S1.

(a)

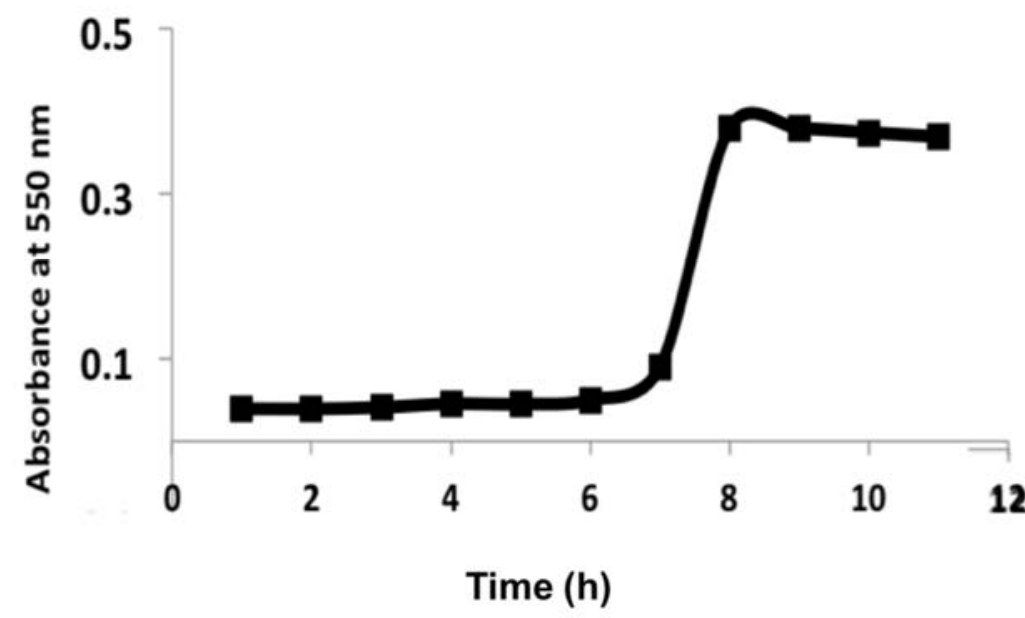

(b)

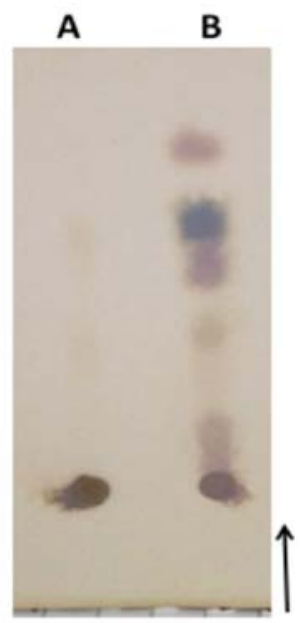

Figure S1. (a) Reducing sugar estimation during hydrolysis of Agp for liberation of oligosaccharides. (b) TLC analysis of hydrolysates of Agp. Lane A- Agp; lane B- hydrolysates of Agp after acid hydrolysis. Arrow represents the direction of TLC run. 


\section{Figure S2}

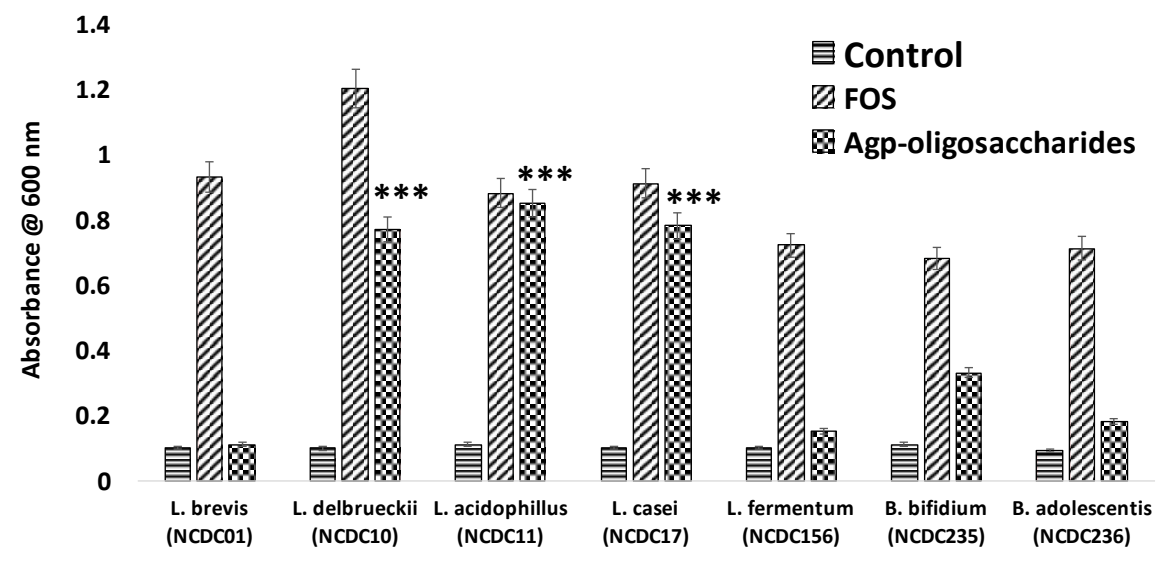

Figure S2. Screening of growth response of species of Lactobacillus and Bifidobacterium using Agp-oligosaccharides $(0.5 \%)$ as sole carbon source. Increase in absorbance of the growth media at $660 \mathrm{~nm}$ was considered as positive response. The incubation time was 48 h. L. delbrueckii, L. acidiphillus and L. casei showed significant growth compared to control/untreated cells. FOS: Fructooligosaccharides. Each sample was tested in triplicates and represented as mean \pm SD. ${ }^{* * *} p<0.05$ compared to control 
Figure S3

(a)

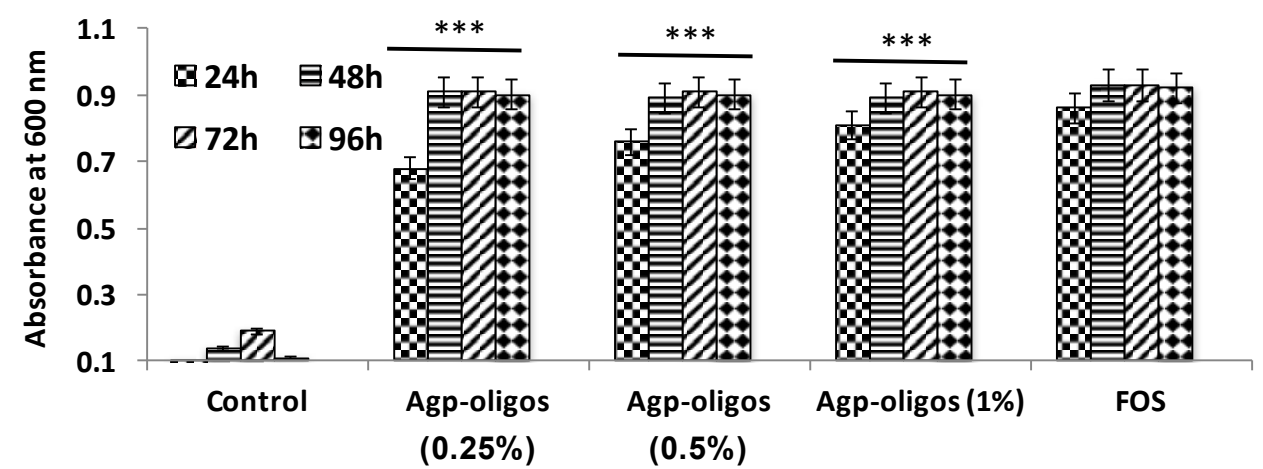

(b)

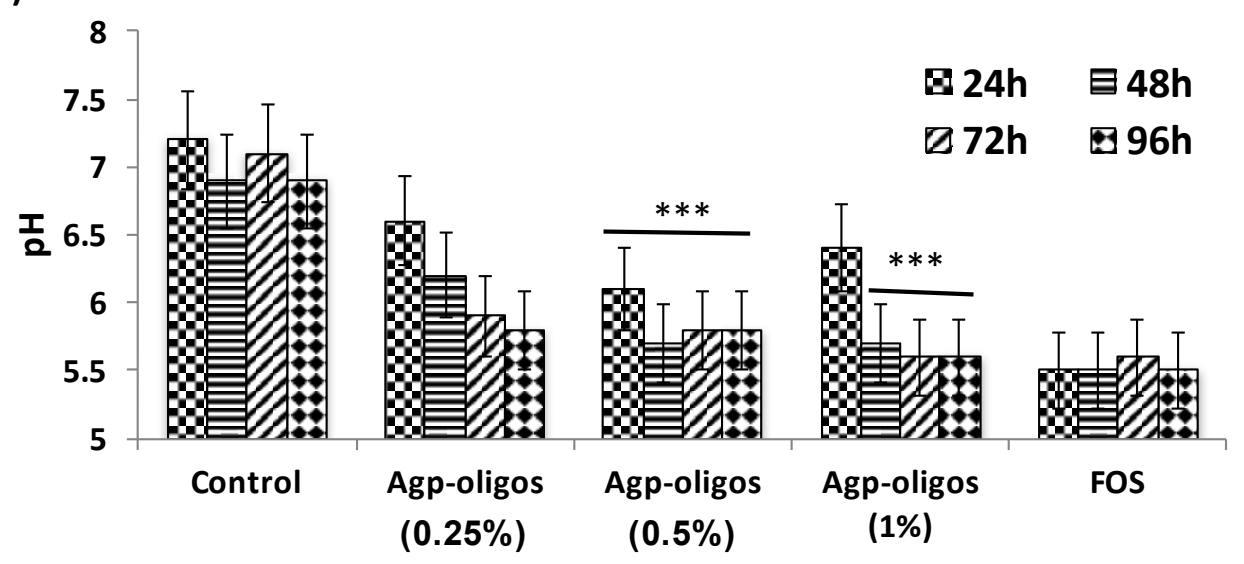

(c)

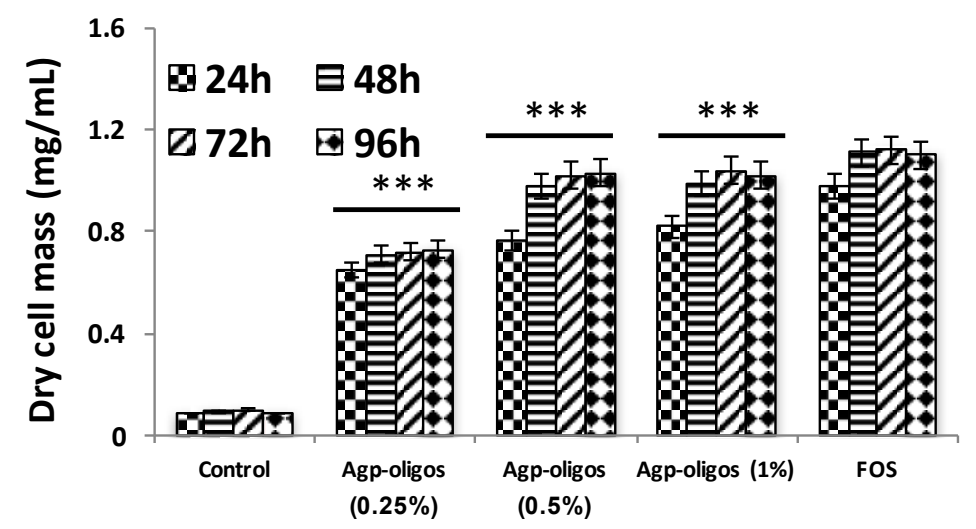

Figure S3. Growth characteristics of L. acidophilus (NCDC 11) with Agpoligosaccharides. Agp-oligosaccharides were metabolized by L. acidophilus. The optimum time was found to be $72 \mathrm{~h}$. L. acidophilus showed maximum growth in the culture broth with $0.5 \%$ test sample or higher. FOS: Fructooligosaccharides. Each sample was tested in triplicates and represented as mean \pm SD. ${ }^{* * *} p<0.05$ compared to control. 
Figure S4.

(a)

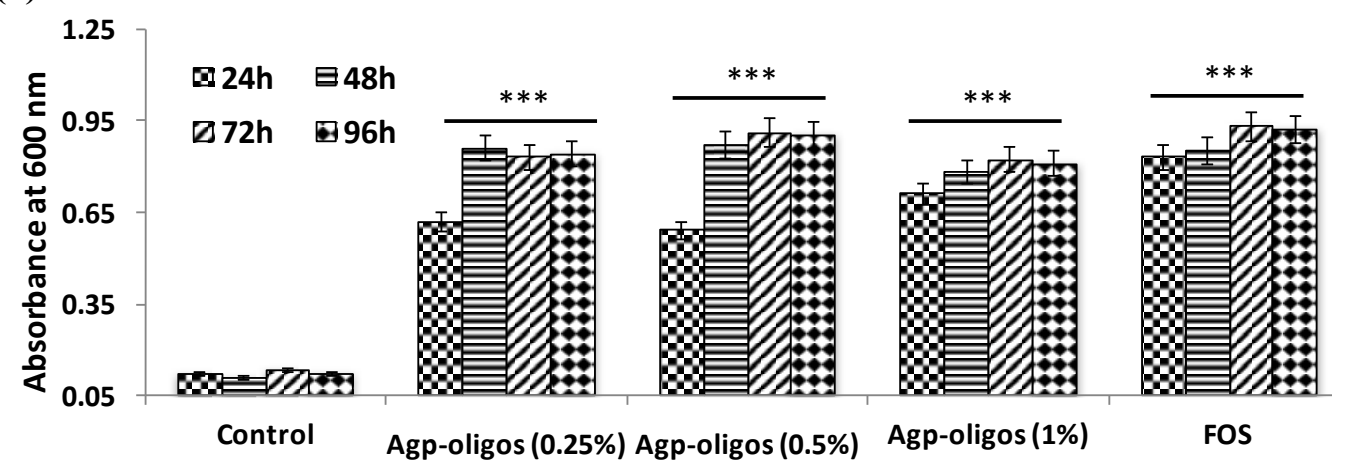

(b)

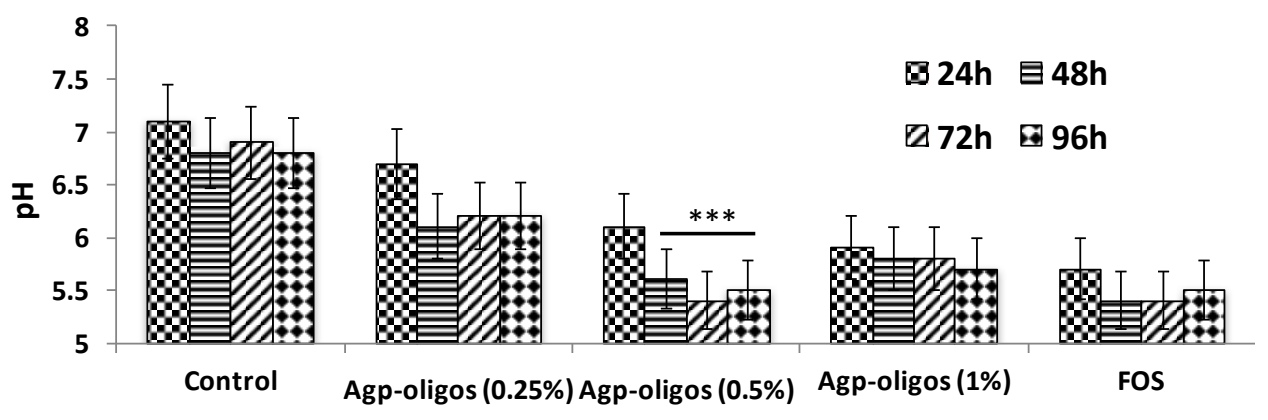

(c)

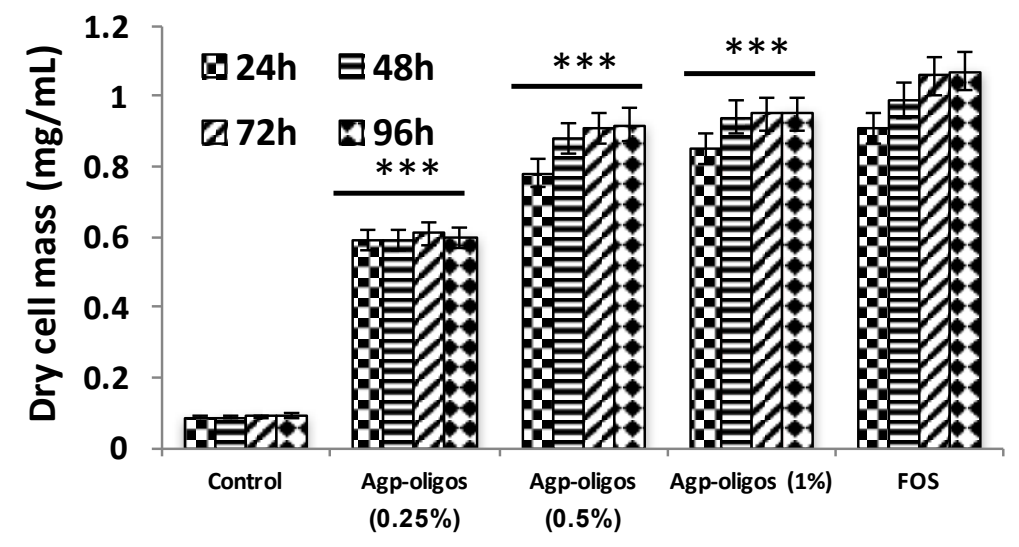

Figure S4. Growth characteristics of L. casei (NCDC 17) with Agp-oligosaccharides. Agp-oligosaccharides were metabolized by $L$. casei. The optimum time was found to be 48 h. L. casei showed maximum growth in the culture broth with $0.5 \%$ test sample or higher. FOS: Fructooligosaccharides. Each sample was tested in triplicates and represented as mean $\pm \mathrm{SD}$. ${ }^{* * *} p<0.05$ compared to control. 
Figure S5

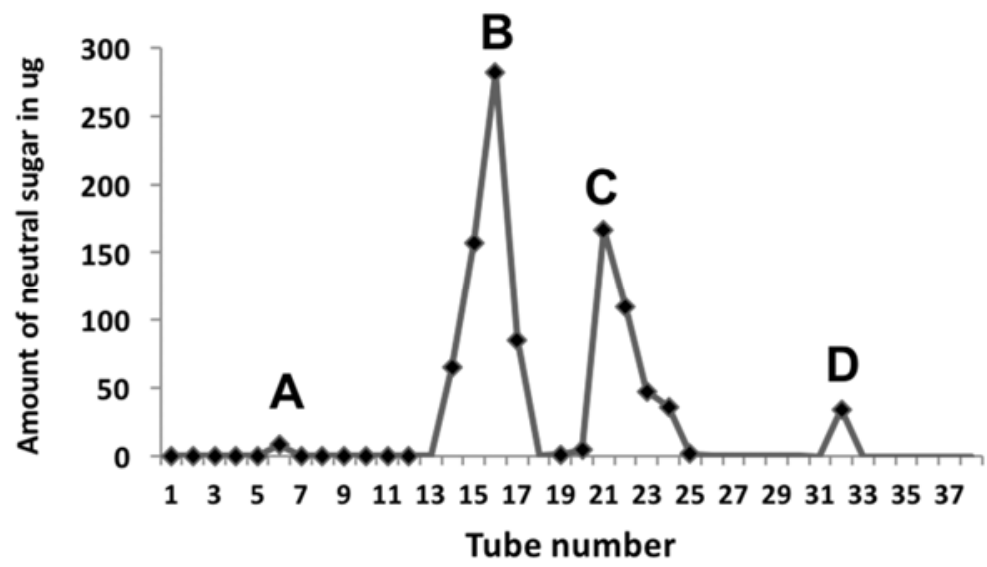

Figure S5. Elution profile of oligosaccharides on Bio Gel P-2. The eluates were measured in terms of neutral sugars using DNS method. Two major peaks are designated as B and C. Peak D is a minor component. The Agp-oligosaccharides were eluted with double distilled water. 


\section{Figure S6}

(a)

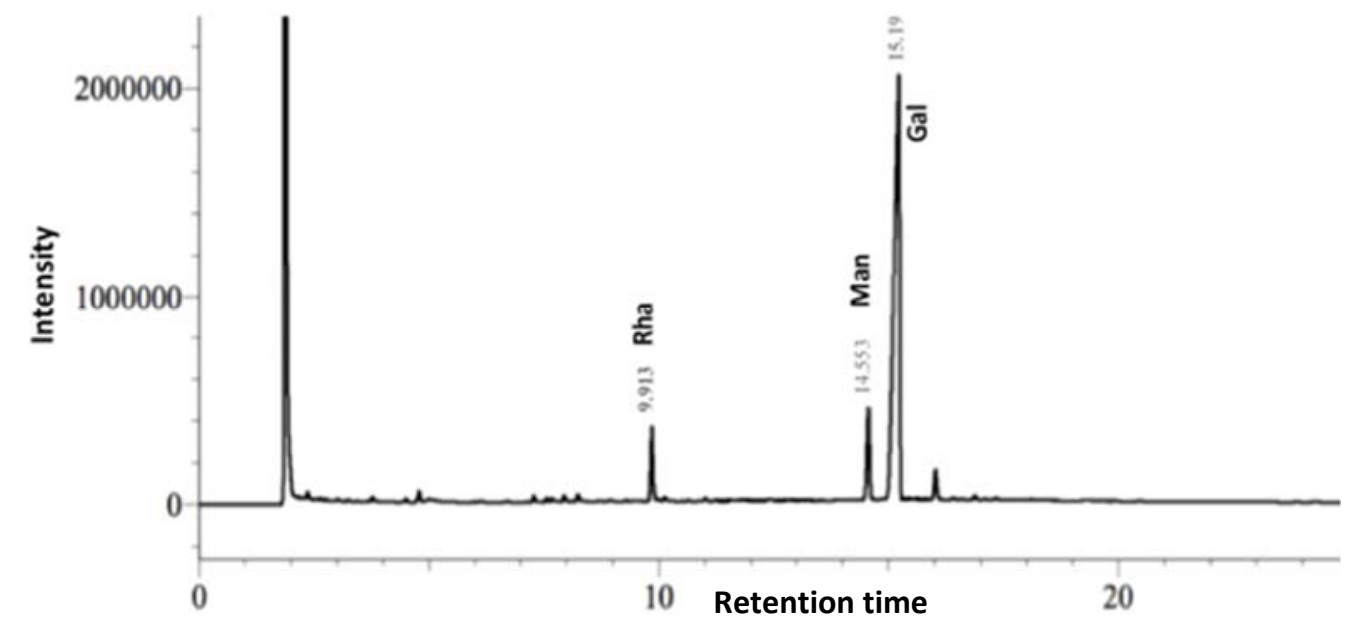

(b)

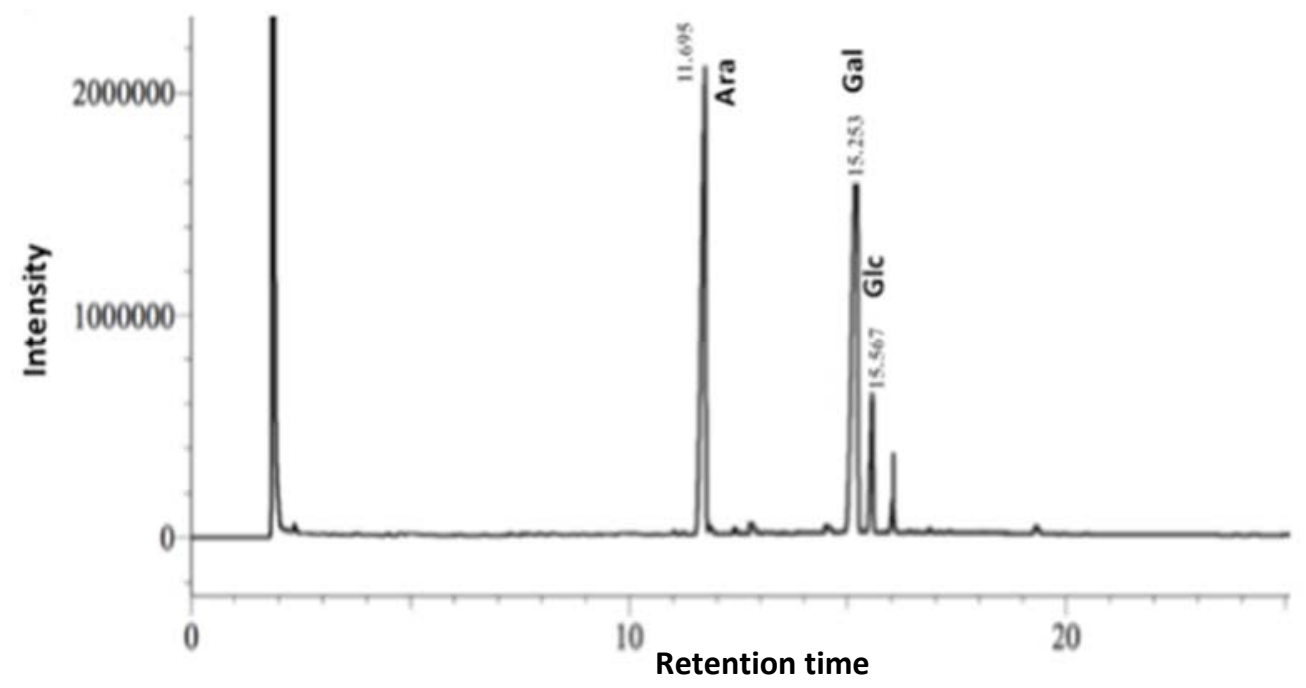

Figure S6. Sugar composition analysis of major Agp-oligosaccharides I and II. (a) Agp-oligosaccharide I is rich with galactose (92.8\%) along with rhamnose $(3.5 \%)$ and mannose (1.8\%). (b) Agp-oligosaccharide II is composed of arabinose (55.6\%) and galactose $(37.5 \%)$ as major sugars and glucose $(4.3 \%)$ as a minor component. 